\title{
POINT EVALUATIONS AND POLYNOMIAL APPROXIMATION IN THE MEAN WITH RESPECT TO HARMONIC MEASURE
}

\author{
JOHN AKEROYD
}

(Communicated by John B. Conway)

\begin{abstract}
For $1 \leq s<\infty$ and crescents ${ }^{1} G$, with harmonic measure $\omega$, the author examines the collection of bounded point evaluations, bpe $\left(P^{s}(\omega)\right)$, (resp. analytic bounded point evaluations, abpe $\left.\left(P^{s}(\omega)\right)\right)$ for polynomials with respect to the $L^{s}(\omega)$ norm. If the polynomials are dense in the generalized Hardy space $H^{s}(G)$, then bpe $\left(P^{s}(\omega)\right)=\operatorname{abpe}\left(P^{s}(\omega)\right)=G$ (Theorem 4). If the polynomials are not dense in $H^{s}(G)$, then (with a mild restriction on $\partial G$ ) $\operatorname{bpe}\left(P^{s}(\omega)\right)=\operatorname{abpe}\left(P^{s}(\omega)\right)=\operatorname{int}\left(\bar{G}^{\wedge}\right)$ (Theorem 7).
\end{abstract}

If $G$ is a crescent, $\omega \equiv \omega\left(\cdot, G, z_{0}\right)$ denotes harmonic measure for $G$ at some $z_{0}$ in $G$, and $1 \leq s<\infty$, then what is the collection of bounded [resp. analytic bounded] point evaluations for polynomials with respect to the $L^{s}(\omega)$ norm? Questions of this sort, phrased in terms of area measure rather than harmonic measure, have been answered by James Brennan [2]. While some of the techniques used in the area measure case have application to our problem, for the most part, new approaches are required in the context of harmonic measure.

1. Notation. If $G$ is a bounded Dirichlet region (open) in the complex plane and $z_{0} \in G$, then let $\mu\left(\cdot, G, z_{0}\right)$ (or $\nu\left(\cdot, G, z_{0}\right.$ ) etc.) denote harmonic measure for $G$ at $z_{0}$.

2. Definition. A crescent is a region (open) in the complex plane bounded by two Jordan curves which intersect in a single point (the multiple boundary point) such that one of the curves is internal to the other.

For any crescent $G$, let $\Omega_{G}$ denote the bounded component of the complement of $\bar{G}$ (the closure of $G$ ), $\partial_{\infty} G=\partial\left(\bar{G}^{\wedge}\right.$ ) and $\partial_{0} G=\partial G \backslash \partial_{\infty} G$ (where $\bar{G}^{\wedge}$ denotes the polynomially convex hull of the closure of $G$ ). Notice that $\partial_{0} G$ is the "inner boundary" of $G$ with the multiple boundary point deleted.

Received by the editors August 31, 1987, and in revised form, January 25, 1988. This paper was presented at the Great Plains Operator Theoretic Seminar (5/9/88), sponsored by Indiana University-Purdue University at Indianapolis.

1980 Mathematics Subject Classification (1985 Revision). Primary 46E15, 46E30; Secondary $47 \mathrm{~B} 38$.

${ }^{1}$ A cresent is a region (open) in the complex plane bounded by two Jordan curves which intersect in a single point such that one of the curves is internal to the other. 
3. Definition. Suppose $\sigma$ is a compactly supported measure in the complex plane, $1 \leq s<\infty, P^{s}(\sigma)$ is the closure of the polynomials in $L^{s}(\sigma)$, and $\|\cdot\|_{\sigma, s} \equiv\left\{\int|\cdot|^{s} d \sigma\right\}^{1 / s}$.

(i) $\lambda \in \operatorname{bpe}\left(P^{s}(\sigma)\right)$ ( $\lambda$ is a bounded point evaluation for $\left.P^{s}(\sigma)\right)$ if there exists a constant $C>0$ such that $|p(\lambda)| \leq C\|p\|_{\sigma, s}$ for all polynomials $p$.

(ii) $\lambda \in \operatorname{abpe}\left(P^{s}(\sigma)\right)\left(\lambda\right.$ is an analytic bounded point evaluation for $\left.P^{s}(\sigma)\right)$ if there exist constants $\varepsilon>0$ and $M>0$ such that $|p(z)| \leq M\|p\|_{\sigma, s}$ for all $z$ in $B(\lambda ; \varepsilon)$ and all polynomials $p$.

Generally speaking, when $G$ is a region in the complex plane and $1 \leq s<\infty$, then $H^{s}(G)$ denotes the collection of all analytic functions $f$ on $G$ for which $|f|^{s}$ has a harmonic majorant on $G$. For $f$ in $H^{s}(G)$ and $z_{0}$ in $G$, let $\|f\|_{\left(G, z_{0}\right)}=u_{f}\left(z_{0}\right)^{1 / s}$, where $u_{f}$ is the least harmonic majorant of $|f|^{s}$ on $G$. It can be shown that $\|\cdot\|_{\left(G, z_{0}\right)}$ defines a norm on $H^{S}(G)$ and under this norm, $H^{s}(G)$ is a Banach space. Moreover, different norming points yield equivalent norms. If $G$ is a crescent, then $H^{s}(G)$ is isometrically isomorphic to $R^{s}(\bar{G}, \omega)$ (the closure in $L^{s}(\omega)$ of the rational functions with poles off $\bar{G}$; $\left.\omega \equiv \omega\left(\cdot, G, z_{0}\right)\right)$.

If $G$ is a crescent, $\omega \equiv \omega\left(\cdot, G, z_{0}\right)$, and $1 \leq s<\infty$, then, depending on $G$, we may have $P^{s}(\omega)=H^{s}(G)$ (density of the polynomials in $H^{s}(G)$ ) or $P^{s}(\omega) \neq H^{s}(G)$ [1]. Our investigation of point evaluations is divided into these two cases.

4. Theorem. Suppose $G$ is a crescent, $\omega \equiv \omega\left(\cdot, G, z_{0}\right)$, and $1 \leq s<\infty$. If $P^{s}(\omega)=H^{s}(G)$, then $\operatorname{bpe}\left(P^{s}(\omega)\right)=\operatorname{abpe}\left(P^{s}(\omega)\right)=G$.

Proof (Sketch). That

$$
G \subseteq \operatorname{abpe}\left(P^{s}(\omega)\right) \subseteq \operatorname{bpe}\left(P^{s}(\omega)\right) \subseteq G \cup \partial_{0} G
$$

follows from general theory. By the Riemann Mapping Theorem and [3, Theorem 3.16], if $\lambda \in \partial_{0} G$ and $0<p<\frac{1}{2}$, then $(z-\lambda)^{-1} \in H^{p}(G)$. Let $m$ be an integer bigger than $2 s$. From our hypothesis, it now follows that $(z-\lambda)^{-1 / m} \in P^{S}(\omega)$. The rest of the argument mimics the proof of [2, Theorem 2] and shows that $\lambda \notin \operatorname{bpe}\left(P^{s}(\omega)\right)$. Hence, $\left\{\partial_{0} G\right\} \cap \operatorname{bpe}\left(P^{s}(\omega)\right)=\varnothing$, and we are done.

The central part of this paper relates to the case $P^{s}(\omega) \neq H^{s}(G)$. That is, if $G$ is a crescent, $\omega \equiv \omega\left(\cdot, G, z_{0}\right), 1 \leq s<\infty$, and $P^{s}(\omega) \neq H^{s}(G)$, then what is bpe $\left(P^{S}(\omega)\right)$ [resp. abpe $\left.\left(P^{S}(\omega)\right)\right]$ ? In particular, which points in $\partial_{0} G$ are also in bpe $\left(P^{S}(\omega)\right)$ [resp. abpe $\left.\left(P^{S}(\omega)\right)\right]$ ? The answer, given in this paper, to the last question, involves a mild restriction on $\partial_{0} G$. Before we explore these questions, some preliminaries are needed. 
Suppose $G$ is a crescent and $\lambda^{\prime}$ and $\lambda^{\prime \prime}$ are distinct points in $\partial_{0} G$. Let $S_{G}\left[\lambda^{\prime}, \lambda^{\prime \prime}\right]=\left\{\gamma: \gamma\right.$ is a Jordan arc with endpoints $\lambda^{\prime}$ and $\lambda^{\prime \prime}$ such that $\gamma \subseteq$ $\left.G \cup\left\{\lambda^{\prime}, \lambda^{\prime \prime}\right\}\right\}, T_{G}\left[\lambda^{\prime}, \lambda^{\prime \prime}\right]=\left\{\Gamma: \Gamma\right.$ is a Jordan curve such that $\left\{\lambda^{\prime}, \lambda^{\prime \prime}\right\} \subseteq \Gamma \subseteq$ $\Omega_{G} \cup\left\{\lambda^{\prime}, \lambda^{\prime \prime}\right\}$ and if $\nu \equiv \nu\left(\cdot, \operatorname{inside}(\Gamma), \xi_{0}\right)$, then $\int \log (\operatorname{dist}(\xi, \bar{G})) d \nu(\xi)>$ $-\infty\}$, and let $\left(\lambda^{\prime}, \lambda^{\prime \prime}\right)_{G}$ denote the relatively open subarc of $\partial_{0} G$ which has endpoints $\lambda^{\prime}$ and $\lambda^{\prime \prime}$.

For any crescent $G$ and any pair of distinct points $\lambda^{\prime}$ and $\lambda^{\prime \prime}$ in $\partial_{0} G$, both $S_{G}\left[\lambda^{\prime}, \lambda^{\prime \prime}\right]$ and $T_{G}\left[\lambda^{\prime}, \lambda^{\prime \prime}\right]$ are nonempty collections. Justification for the nontriviality of $T_{G}\left[\lambda^{\prime}, \lambda^{\prime \prime}\right]$ is provided in the appendix.

5. Definition. Suppose $G$ is a crescent and $\lambda^{\prime} \in \partial_{0} G$. Let $\Theta_{G}\left(\lambda^{\prime}\right)$ be the collection of points $\lambda^{\prime \prime}$ in $\partial_{0} G, \lambda^{\prime \prime} \neq \lambda^{\prime}$, for which there exists $\gamma$ in $S_{G}\left[\lambda^{\prime}, \lambda^{\prime \prime}\right], \Gamma$ in $T_{G}\left[\lambda^{\prime}, \lambda^{\prime \prime}\right]$, and a positive constant $c$ such that if $F=\gamma \cup \Gamma$, $V=\operatorname{inside}(\Gamma), W=\operatorname{interior}\left(F^{\wedge} \backslash \Gamma^{\wedge}\right), G_{1}$ and $G_{2}$ are the components of $G \backslash \gamma, \nu \equiv \nu\left(\cdot, V, \xi_{0}\right), \mu \equiv \mu\left(\cdot, W, \eta_{0}\right)$, and $\rho \equiv \rho_{1}\left(\cdot, G_{1}, z_{1}\right)+\rho_{2}\left(\cdot, G_{2}, z_{2}\right)$, then

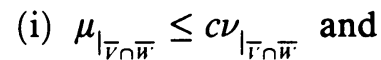

(ii) $\int_{\gamma} \log (d \rho / d \mu) d \mu>-\infty$.

One should observe that (in Definition 5) condition (i) can be satisfied in the absence of condition (ii) and conversely. Therefore, $\boldsymbol{\Theta}_{G}\left(\lambda^{\prime}\right)$ is limited only by the extent to which conditions (i) and (ii) cannot be satisfied simultaneously.

It would be nice to geometrically characterize what it means for $\lambda^{\prime \prime}$ to be in $\Theta_{G}\left(\lambda^{\prime}\right)$, but this is no easy task. However, if one is willing to sacrifice precision, conditions which easily imply that $\lambda^{\prime \prime} \in \Theta_{G}\left(\lambda^{\prime}\right)$ are not hard to find. For example, suppose $\varepsilon$ and $\phi$ are positive constants, $\lambda \in \mathbf{C}$ (the complex plane), and let

$$
\begin{aligned}
& A(\lambda, \varepsilon, \phi)=\left\{e^{i \phi} z+\lambda: 0<|z|<\varepsilon \text { and either }-\left(\frac{\pi}{4}+\varepsilon\right)<\arg (z)<\left(\frac{\pi}{4}+\varepsilon\right)\right. \\
&\left.\quad \text { or }-\left(\frac{\pi}{4}+\varepsilon\right)<\arg (-z)<\left(\frac{\pi}{4}+\varepsilon\right)\right\}, \\
& B(\lambda, \varepsilon, \phi)=\left\{e^{i \phi} z+\lambda:|z-\varepsilon|<\varepsilon\right\}, \\
& C(\lambda, \varepsilon, \phi)=\left\{e^{i \phi} z+\lambda: 0<|z|<\varepsilon \text { and }-\varepsilon<\arg (z)<\varepsilon\right\} .
\end{aligned}
$$

If $\lambda^{\prime}$ and $\lambda^{\prime \prime}$ are distinct points in $\partial_{0} G$, then either of the following two conditions imply that $\lambda^{\prime \prime} \in \boldsymbol{\Theta}_{G}\left(\lambda^{\prime}\right)$ :

(a) there exist positive constants $\varepsilon^{\prime}, \varepsilon^{\prime \prime}, \phi^{\prime}$ and $\phi^{\prime \prime}$ such that $\left\{\partial_{0} G\right\} \cap$ $A\left(\lambda^{\prime}, \varepsilon^{\prime}, \phi^{\prime}\right)=\varnothing=\left\{\partial_{0} G\right\} \cap A\left(\lambda^{\prime \prime}, \varepsilon^{\prime \prime}, \phi^{\prime \prime}\right)$,

(b) there exist positive constants $\varepsilon^{\prime}, \varepsilon^{\prime \prime}, \phi_{1}^{\prime}, \phi_{2}^{\prime}, \phi_{1}^{\prime \prime}$ and $\phi_{2}^{\prime \prime}$ such that $B\left(\lambda^{\prime}, \varepsilon^{\prime}, \phi_{1}^{\prime}\right) \cup B\left(\lambda^{\prime \prime}, \varepsilon^{\prime \prime}, \phi_{1}^{\prime \prime}\right) \subseteq \Omega_{G}$ and $C\left(\lambda^{\prime}, \varepsilon^{\prime}, \phi_{2}^{\prime}\right) \cup C\left(\lambda^{\prime \prime}, \varepsilon^{\prime \prime}, \phi_{2}^{\prime \prime}\right) \subseteq$ $G$.

With a little more work, other variations of the "double-cone" condition given in (a) are shown to imply that $\lambda^{\prime \prime} \in \Theta_{G}\left(\lambda^{\prime}\right)$. Furthermore, it should be noted 
that, for any crescent $G$, the set of all $\lambda$ in $\partial_{0} G$ for which there exist $\varepsilon$ and $\phi$ such that $B(\lambda, \varepsilon, \phi) \subseteq \Omega_{G}$ is dense in $\partial_{0} G$.

Are there crescents $G$ such that $\Theta_{G}\left(\lambda^{\prime}\right)=\varnothing$ for all $\lambda^{\prime}$ in $\partial_{0} G$ ? If so, then such crescents should be considered unusual. To insist that $\Theta_{G}\left(\lambda^{\prime}\right) \neq \varnothing$, for some $\lambda^{\prime}$ in $\partial_{0} G$, is far less restrictive than requiring that $\partial_{0} G$ be somewhere smooth or rectifiable, as is evident from our sufficient condition (a).

6. Lemma. Suppose $G$ is a crescent, $\omega \equiv \omega\left(\cdot, G, z_{0}\right), 1 \leq s<\infty$, and $\lambda^{\prime}$ and $\lambda^{\prime \prime}$ are distinct points in $\partial_{0} G$. If $\left\{q_{n}\right\}$ is a sequence of polynomials such that $\int\left|q_{n}\right|^{s} d \omega \leq C<\infty$ (for all $n$ ), $\gamma \in S_{G}\left[\lambda^{\prime}, \lambda^{\prime \prime}\right], \rho$ is as in Definition 5 , and $\mu$ is a Borel measure such that $0<\mu(\gamma)<\infty, \rho \ll \mu$ (on $\gamma$ ) and $\int_{\gamma} \log (d \rho / d \mu) d \mu>-\infty$, then $\int_{\gamma} \log \left|q_{n}\right| d \mu \leq M<\infty$ (for all $\left.n\right)$.

Proof. By the definition of $\omega$ and $\rho$, the subharmonicity of $\left|q_{n}\right|^{s}$ and our hypothesis, there exists $R$ such that $\int_{\gamma}\left|q_{n}\right| d \rho \leq R<\infty$ (for all $n$ ). Hence,

$$
\int_{\gamma}\left|q_{n}\right|\left(\frac{d \rho}{d \mu}\right) \frac{d \mu}{\mu(\gamma)} \leq \frac{R}{\mu(\gamma)} \quad(\text { for all } n) .
$$

Jensen's Inequality implies that

$$
\int_{\gamma}\left(\log \left|q_{n}\right|+\log \left(\frac{d \rho}{d \mu}\right)\right) d \mu \leq \mu(\gamma)(\log (R)-\log (\mu(\gamma))) .
$$

Since $\int_{\gamma} \log (d \rho / d \mu) d \mu>-\infty$, the result follows.

7. Theorem. Suppose $G$ is a crescent, $\omega \equiv \omega\left(\cdot, G, z_{0}\right)$, and $1 \leq s<\infty$. If $P^{s}(\omega) \neq H^{s}(G)$ and $\lambda^{\prime} \in \partial_{0} G$, then

(i) $G \cup \Omega_{G} \subset \operatorname{abpe}\left(P^{s}(\omega)\right) \subset \operatorname{bpe}\left(P^{s}(\omega)\right) \subset \operatorname{int}\left(\bar{G}^{\wedge}\right)$, and

(ii) for any $\lambda^{\prime \prime}$ in $\boldsymbol{\Theta}_{G}\left(\lambda^{\prime}\right),\left(\lambda, \lambda^{\prime \prime}\right)_{G} \subset \operatorname{abpe}\left(P^{s}(\omega)\right)$.

Proof. The proof of (i) is quite commonplace and therefore is omitted. Our strategy for the proof of (ii) is to assume that there exists $\lambda$ in $\left(\lambda^{\prime}, \lambda^{\prime \prime}\right)_{G}$ such that $\lambda \notin \operatorname{abpe}\left(P^{s}(\omega)\right)$ and show that this forces us to conclude that $P^{s}(\omega)=$ $H^{s}(G)$.

If $\lambda \in\left(\lambda^{\prime}, \lambda^{\prime \prime}\right)_{G}$ and $\lambda \notin \operatorname{abpe}\left(P^{s}(\omega)\right)$, then there exists a sequence of polynomials $\left\{p_{n}\right\}$ such that $\left\|p_{n}\right\|_{\omega, s}=1$ (for all $n$ ) and $\left\|(z-\lambda) p_{n}\right\|_{\omega, s} \rightarrow 0$, as $n \rightarrow \infty$. Therefore, since harmonic measure has no point masses, there exists a sequence of points $\left\{z_{n}\right\}$ such that $z_{n} \rightarrow \lambda$ and $\left|p_{n}\left(z_{n}\right)\right| \rightarrow \infty$, as $n \rightarrow \infty$.

Now $\lambda^{\prime \prime} \in \Theta_{G}\left(\lambda^{\prime}\right)$, and so there exist a Jordan curve $\Gamma$ and a Jordan arc $\gamma$ which satisfy the requirements of Definition 5. As before, let $F=\gamma \cup \Gamma$, $V=\operatorname{inside}(\Gamma), W=\operatorname{interior}\left(F^{\wedge} \backslash \Gamma^{\wedge}\right), \nu \equiv \nu\left(\cdot, V, \xi_{0}\right)$, and $\mu \equiv \mu\left(\cdot, W, \eta_{0}\right)$. Since $z_{n} \rightarrow \lambda \in W$, we may assume that $\left\{z_{n}\right\} \subset W$.

Choose Jordan subarcs $\gamma_{1}$ and $\gamma_{2}$ of $\Gamma \cap$ interior $\left(F^{\wedge}\right)$ such that $\gamma_{2}^{0} \subset \gamma_{2} \subset$ $\gamma_{1}^{0} \subset \gamma_{1}\left(\subset \Omega_{G}\right)$, where $\gamma_{i}^{0}$ denotes $\gamma_{i}$ with endpoints deleted (for $\left.i=1,2\right)$. Let $E=V \cup W \cup\left\{\gamma_{2}^{0}\right\}, \sigma \equiv \sigma(\cdot, E, \lambda)$ and $\sigma_{n} \equiv \sigma_{n}\left(\cdot, E, z_{n}\right)$. By Definition 5, 
and properties of harmonic measure and conformal maps, there exists a positive constant $k$ such that,

$$
\left\{\begin{array}{l}
\frac{1}{k} \sigma \leq \sigma_{n} \leq k \sigma \quad(\text { for all } n), \\
\frac{1}{k} \mu_{\mid \gamma} \leq \sigma_{\mid \gamma} \leq k \mu_{\mid \gamma}, \\
\frac{1}{k} \nu_{\mid \Gamma \backslash \gamma_{1}} \leq \sigma_{\mid \Gamma \backslash \gamma_{1}} \leq k \nu_{\mid \Gamma \backslash \gamma_{1}} .
\end{array}\right.
$$

Since $\log \left|p_{n}\right|$ is subharmonic, the definition of $\sigma_{n}$ implies that $\int \log \left|p_{n}\right| d \sigma_{n}$ $\geq \log \left|p_{n}\left(z_{n}\right)\right| \rightarrow \infty$, as $n \rightarrow \infty$. Moreover, from Definition 5, (7.1), and the proof of Lemma 6, theré exists a constant $M$ such that $\int_{\gamma} \log \left|p_{n}\right| d \sigma_{n} \leq M<\infty$ (for all $n$ ). Therefore,

$$
\int_{\Gamma \backslash \gamma_{2}^{0}} \log \left|p_{n}\right| d \sigma_{n} \rightarrow \infty, \quad \text { as } n \rightarrow \infty .
$$

Now choose $g$ in $L^{s}(\omega)^{*}\left(=L^{t}(\omega) ; \frac{1}{s}+\frac{1}{t}=1\right)$ such that $g \perp P^{s}(\omega)$. For $\xi$ in $\mathbf{C} \backslash \partial G$, let

$$
\hat{g}(\xi)=\int \frac{g(z)}{z-\xi} d \omega(z)
$$

If $\xi \in \Omega_{G}$, then

$$
p_{n}(\xi) \hat{g}(\xi)=\int \frac{p_{n}(z) g(z)}{z-\xi} d \omega(z) .
$$

Therefore, if $\xi \in \Omega_{G}$ and $p_{n}(\xi) \neq 0$, then

$$
\hat{g}(\xi)=\frac{1}{p_{n}(\xi)} \int \frac{p_{n}(z) g(z)}{z-\xi} d \omega(z) .
$$

We may assume that $p_{n}(\xi) \neq 0$ for all $\xi$ in $\Gamma$ and $n=1,2,3, \ldots$.

Because $\Gamma \in T_{G}\left[\lambda^{\prime}, \lambda^{\prime \prime}\right]$ and by (7.1), there is a positive constant $C$ such that

$$
\begin{aligned}
& \int_{\Gamma \backslash \gamma_{2}^{0}} \log \left|\int \frac{p_{n}(z) g(z)}{z-\xi} d \omega(z)\right| d \sigma_{n}(\xi) \\
& \quad \leq \int_{\Gamma \backslash y_{2}^{0}}\left(\log \left(\left\|p_{n}\right\|_{\omega, s}\|g\|_{\omega, t}\right)-\log (\operatorname{dist}(\xi, \bar{G}))\right) d \sigma_{n}(\xi) \quad\left(\frac{1}{s}+\frac{1}{t}=1\right) \\
& \quad \leq C<\infty,
\end{aligned}
$$

for all $n$. Consequently, from (7.2) and (7.3) it is evident that

$$
\int_{\Gamma \backslash y_{2}^{0}} \log |\hat{g}(\xi)| d \sigma_{n}(\xi) \leq C-\int_{\Gamma \backslash \gamma_{2}^{0}} \log \left|p_{n}(\xi)\right| d \sigma_{n}(\xi) \rightarrow-\infty, \quad \text { as } n \rightarrow \infty .
$$

Notice that by our choice of $\Gamma, \log ^{+}|\hat{g}(\xi)| \in L^{1}(\nu)$. So, by (7.1), (7.4), and the fact that $\sigma_{n} \rightarrow \sigma$ wk $^{*}($ as $n \rightarrow \infty)$, we have

$$
\int_{\Gamma \backslash \gamma_{2}^{0}} \log |\hat{g}(\xi)| d \sigma(\xi)=-\infty
$$


Since $\hat{g}$ is analytic in $\Omega_{G}, \gamma_{1}$ is a compact subset of $\Omega_{G}$ and $\log ^{+}|g(\xi)| \epsilon$ $L^{1}(\nu)$, we may combine (7.1), (7.5), and [4, Theorem 3.2 (Chapter 5)] to get that

$$
\log \left|\hat{g}\left(\xi_{0}\right)\right| \leq \int \log |\hat{g}(\xi)| d \nu(\xi)=-\infty .
$$

Hence, $\hat{g}\left(\xi_{0}\right)=0$. Since $\xi_{0}$ is arbitrary in $V$, we conclude that $\hat{g}_{\mid V} \equiv 0$. Therefore, by the analyticity of $\hat{g}$ in $\Omega_{G}, \hat{g}_{\mid \Omega_{G}} \equiv 0$. This, coupled with the fact that $g \perp P^{s}(\omega)$, means that $g \perp H^{s}(G) \quad\left(\simeq R^{s}(\bar{G}, \omega)\right)$.

In summary, with the assumption that there exists $\lambda$ in $\left(\lambda^{\prime}, \lambda^{\prime \prime}\right)_{G}$ such that $\lambda \notin \operatorname{abpe}\left(P^{s}(\omega)\right)$, we have shown that if $g \in L^{s}(\omega)^{*}$ and $g \perp P^{s}(\omega)$, then $g \perp H^{s}(G)$. So, by the Hahn-Banach Theorem, $P^{s}(\omega)=H^{s}(G)$. The result now follows.

\section{APPENDIX}

From the following proposition we shall conclude that $T_{G}\left[\lambda^{\prime}, \lambda^{\prime \prime}\right]$ (as defined in this paper) is nonempty; but first some notation. For constants $r$ and $R$, $0<r<R$, let $\mathbf{D}_{r}=\{z:|z|<r\}, \mathbf{D} \equiv \mathbf{D}_{1}, A(r, R)=\{z: r \leq|z|<R\}$, and $H_{r}=\{z:-r<\operatorname{Im}(z)<r\}$.

Proposition. Suppose $g$ is a continuous complex-valued function defined on $\mathbf{D}$, and $\alpha$ and $\beta$ are distinct points in $\partial \mathbf{D}$. Then there exists a Jordan curve $\Gamma$ such that $\{\alpha, \beta\} \subseteq \Gamma \subseteq \mathbf{D} \cup\{\alpha, \beta\}$ and if $\nu \equiv \nu\left(\cdot, \operatorname{inside}(\Gamma), \xi_{0}\right)$, then $g \in L^{1}(\nu)$.

Proof. Let us assume that $\alpha=-1$ and $\beta=1$. The general case follows by composing $g$ with an appropriate Möbius transformation. For positive integers $n$, let $r_{n}=1-2^{-n}$ and $M_{n}=1+\sup \left\{|g(z)|: z \in \mathbf{D}_{r_{n}}\right\}$. Choose a sequence $\left\{t_{n}\right\}$ of positive numbers, $\frac{1}{2}>t_{1}>t_{2}>\cdots>t_{n} \rightarrow 0($ as $n \rightarrow \infty)$, such that if $S_{n}=\bigcup_{k=1}^{n}\left\{\mathbf{D}_{r_{k}} \cap H_{t_{k}}\right\}$ and $\nu_{n} \equiv \nu_{n}\left(\cdot, S_{n}, 0\right)$ then

$$
\nu_{n}\left(\left\{\partial \mathbf{D}_{r_{n}}\right\} \cap H_{t_{n}}\right)<\left(2^{n} M_{n+1}\right)^{-1} \text {, for } n=1,2,3, \ldots \text {. }
$$

Let $S=\bigcup_{n=1}^{\infty} S_{n}, \Gamma=\partial S$, and $\nu \equiv \nu(\cdot, S, 0)$. By the Maximum Principle,

$$
\nu\left(\{\partial S\} \cap A\left(r_{n}, r_{n+1}\right)\right)<\nu\left(\{\partial S\} \backslash \mathbf{D}_{r_{n}}\right) \leq \nu_{n}\left(\left\{\partial \mathbf{D}_{r_{n}}\right\} \cap H_{t_{n}}\right)<\left(2^{n} M_{n+1}\right)^{-1} .
$$

Hence,

$$
\begin{aligned}
\int|g| d \nu & =\int_{\{\partial S\} \cap \mathbf{D}_{r_{1}}}|g| d \nu+\sum_{n=1}^{\infty} \int_{\{\partial S\} \cap A\left(r_{n}, r_{n+1}\right)}|g| d \nu \\
& <\int_{\{\partial S\} \cap \mathbf{D}_{r_{1}}}|g| d \nu+1 \\
& <\infty . \quad \square
\end{aligned}
$$

If $G$ is a crescent, then $\xi \mapsto \log (\operatorname{dist}(\xi, \bar{G}))$ is continuous on $\Omega_{G}$. Let $\phi$ be a Riemann map from $\mathbf{D}$ onto $\Omega_{G}$. Given distinct points $\lambda^{\prime}$ and $\lambda^{\prime \prime}$ in $\partial_{0} G$, choose $\Gamma$ as in the preceding proposition for $\alpha=\phi^{-1}\left(\lambda^{\prime}\right), \beta=\phi^{-1}\left(\lambda^{\prime \prime}\right)$ and 
$g(z)=\log (\operatorname{dist}(\phi(z), \bar{G}))$. From the preceding proposition and properties of harmonic measure and conformal maps, it is evident that $\phi(\Gamma) \in T_{G}\left[\lambda^{\prime}, \lambda^{\prime \prime}\right]$.

\section{ACKNOWLEDGMENT}

Many thanks to both J. B. Conway and the referee for their helpful suggestions.

\section{REFERENCES}

1. J. Akeroyd, Polynomial approximation in the mean with respect to harmonic measure on crescents, Trans. Amer. Math. Soc. 303 (1987), 193-199.

2. J. Brennan, Point evaluations, invariant subspaces and approximation in the mean by polynomials, J. Funct. Anal. 34 (1979), 407-420.

3. Peter L. Duren, Theory of $H^{p}$-spaces, Academic Press, New York, 1970.

4. T. W. Gamelin, Uniform algebras, Chelsea, New York, 1984.

Department of Mathematics, University of Arkansas, Fayetteville, ARkansas 72701 